\title{
Métodos de biópsia pulmonar empregados no Hospital Universitário de Santa Maria: acurácia, sensibilidade e especificidade.
}

Ana Elize Barin ${ }^{1}$; Tamires Tolazzi Martins² ; Antônio Aurelio da Silveira Codevilla ${ }^{3}$; Léo Canterle Dal Osto ${ }^{4}$; Filipe Rafael Stuker ${ }^{5}$ e Melissa Orlandin Premaor6.

\section{Resumo}

INTRODUÇÃO: O uso de biópsias pulmonares como ferramenta diagnóstica é muito comum na prática clínica. Entretanto a acurácia reportada nos estudos parece ser maior do que a encontrada na realidade. $\mathrm{O}$ objetivo desse estudo foi definir a acurácia diagnóstica, sensibilidade e especificidade dos dois principais métodos de biópsia pulmonar empregados no Hospital Universitário de Santa Maria (HUSM): biópsia endobrônquica e biópsia pulmonar percutânea transtorácica (BPPT) guiada por tomografia computadorizada.

MÉTODOS: Foi delineado um estudo transversal em que se analisaram laudos anatomopatológicos das biópsias pulmonares realizadas no HUSM entre os anos de 2003 a 2013. Foram incluídos os laudos que possuíam pelo menos uma modalidade de biópsia pulmonar realizada, sendo precedente ao procedimento cirúrgico, o qual foi considerado como padrãoouro.

RESULTADOS: A acurácia da biópsia realizada por método endobrônquico, aferida através de curva ROC teve como área sob a curva o valor calculado de 0,645. Sua sensibilidade e especificidade foram, respectivamente, $43 \%$ e $85 \%$. A acurácia da BPPT apresentou área sob a 
curva calculada em 0,681. Sua sensibilidade foi $61 \%$ e sua especificidade $75 \%$.

CONCLUSÃO: A acurácia diagnóstica da biópsia endobrônquica e da BPPT encontradas em nosso estudo parecem ser menores do que as reportadas na literatura. Esse fato pode ser devido a diferenças de população e critérios de indicação das biópsias. Mais estudos se fazem necessários para o melhor entendimento desse problema.

Palavras Chave: biópsia; acurácia; pulmonar

Graduanda do curso de Medicina da Universidade Federal de Santa Maria (UFSM) e membro do grupo de pesquisa de Doenças Endócrino-Metabólicas Prevalentes; anaelizebarin@gmail.com

${ }^{2}$ Graduanda do curso de Medicina da Universidade Federal de Santa Maria (UFSM), membro do grupo de pesquisa de Doenças Endócrino-Metabólicas Prevalentes e bolsista do Programa de Iniciação Científica para o Hospital Universitário de Santa Maria PROIC-HUSM; tamitolazzi@gmail.com

${ }^{3}$ Graduando do curso de Medicina da Universidade Federal de Santa Maria (UFSM) e membro do grupo de pesquisa de Doenças Endócrino-Metabólicas Prevalentes. antoniocodevilla@gmail.com

${ }^{4}$ Graduando do curso de Medicina da Universidade Federal de Santa Maria (UFSM), membro do grupo de pesquisa de Doenças Endócrino-Metabólicas Prevalentes e bolsista do Programa de Iniciação Científica para o Hospital Universitário de Santa Maria PROIC-HUSM; leodalosto@live.com

${ }^{5}$ Mestrando em Ciências da Saúde da Universidade Federal de Santa Maria (UFSM) e membro do grupo de pesquisa de Doenças Endócrino-Metabólicas Prevalentes.

${ }^{6}$ Pesquisadora do grupo de pesquisa de Doenças Endócrino-Metabólicas Prevalentes e Orientadora do Programa de Iniciação Científica para o Hospital Universitário de Santa Maria-PROIC-HUSM. premaor@ufsm.com.br 